\title{
Formación identitaria como eje articulador de permanencia y éxito académico de estudiantes de educación superior
}

\author{
Formação identitária como eixo articulador de permanencia e éxito acadêmico \\ de estudantes de educação superior
}

\section{Identity formation as the linchpin of permanence and student academic success in higher education}

\author{
Formation de l'identité comme la cheville ouvrière de la permanence et au succès \\ scolaire dans l'enseignement supérieur \\ Carlos Mejías Sandia* \\ (camejias@utalca.cl)
}

Recebido em 28/11/2014, revisado e aprovado em 15/06/2015; aceito em 20/11/2015

DOI: http://dx.doi.org/10.20435/1984-042X-2016-v.17-n.3(10)

\begin{abstract}
Resumen: Las causas de la deserción y el fracaso académico originan variadas interrogantes que se desprenden del estudio del ingreso o de la deserción universitaria más bien desde lo cuantitativo. Sin embargo, ellas no dan cuenta de la problemática desde los propios actores ni de cómo la variable formación identitaria participa en este proceso y configura el conjunto de representaciones de las/os estudiantes y cómo estos influyen en la complejidad de conjugar factores sociales, motivacionales e institucionales.
\end{abstract}

Palabras clave: identidad; aprendizaje; formación integral.

Resumo: As causas da deserção e o fracasso acadêmico são a fonte de muitas perguntas que se desprendem das pesquisas sobre a deserção universitária, mas fundamentalmente de pesquisas de caráter quantitativo. Porém, estas questões não conseguem dar conta da problemática considerando os atores mesmos, tampouco mostrar como é que a formação identitária participa no processo de ensino e como ela configura o conjunto das representações dos estudantes, e também de como influencia na complexidade de conjugar os fatores sociais, motivacionais e institucionais. Palavras-chave: identidade; aprendizagem; formação integral.

Abstract: The causes of academic failure and dropout varied questions that arise from the study of income or college dropout rather from the quantitative. However, they do not realize the problem from the actors themselves or how varying the identity formation involved in this process and configures the set of representations of the / o students and how these influence the complexity of combining social, and motivational factors institutional.

Key words: identity; learning; comprehensive training.

Résumé: Les causes de l'échec scolaire et le décrochage diverses questions qui découlent de l'étude de revenu ou plutôt College Dropout du quantitatif. Cependant, ils ne se rendent pas compte du problème des acteurs eux-mêmes ou comment la formation variable d'identité impliqué dans ce processus et configure l'ensemble des représentations des élèves / o et comment ceux-ci influent sur la complexité de la combinaison de facteurs sociaux, de motivation et institutionnel.

Mots-clés: identité; l'apprentissage; la formation complète.

\section{ANTECEDENTES}

El alto grado de competitividad, la necesidad de éxito, la presión por obtener un título profesional en busca de mejores ingresos, revela la situación de encrucijada que se les plantea a las y los estudiantes y a sus familias en la actualidad: encrucijada en lo relativo al papel que dicha familia juega, así como la universidad y los propios y propias estudiantes, encrucijada que se materializa

\footnotetext{
* Universidad de Talca, Maule Region, Chile.
} 
respecto a la continuidad educativa y a la inserción socio-laboral, en definitiva, encrucijada frente a la presión de las transformaciones socioculturales.

Esto provoca que el éxito académico se convierta en una variable esencial para la vida profesional y personal. Un número no menor de investigaciones han establecido que para logra dicho éxito se requiere de un alto grado de adhesión a los fines, los medios y los valores de la institución educativa, que probablemente no todos los estudiantes presentan. Aunque no faltan los que aceptan incondicionalmente el proyecto de vida que les ofrece la Institución, es posible que un sector lo rechace, y otro, tal vez el más sustancial, sólo se identifica con el mismo de manera circunstancial. Aceptan, por ejemplo, la promesa de movilidad social y emplean la institución de educación superior para alcanzarla, pero no se identifican con la cultura y los valores que ella promueve, por lo que mantienen hacia la Institución una actitud de acomodo, la cual consiste en transitar por ella con sólo el esfuerzo necesario. $\mathrm{O}$ bien se encuentran con ella en su medio cultural natural pero no creen o no necesitan creer en sus promesas, porque han decidido renunciar a lo que se les ofrece, o lo tienen asegurado de todos modos por su condición social y entonces procuran disociarse de sus exigencias.

Sin embargo, al momento de buscar las causas del fracaso académico se tiende a argüir hacia la calidad de los programas de estudio, la masificación de estudiantes en las aulas, la falta de recursos de las instituciones y al papel de los padres.

Por su parte, los profesores, incentivados o presionados por las instituciones de educación, intentan buscar estrategias motivacionales para tratar de minimizar el fracaso académico desarrollando tipos particulares de motivación de sus estudiantes, entre los que se incluyen: la planeación, concentración en la(s) meta(s), consciencia metacognoscitiva de lo que se pretende aprender y cómo se pretende aprenderlo, búsqueda activa de nueva información, percepciones claras de la retroalimentación, elogio y satisfacción por el logro y ninguna ansiedad o temor al fracaso, todas estrategias formales que no apunta al espacio personal del estudiante ni a sus propias expectativas, miedos, incertidumbres, conocimientos previos, valores y esperanzas.

Esta investigación sobre cómo la formación identitaria de las estudiantes y los estudiantes influye en la retención y éxito académico intenta aportar nuevos antecedentes que permitan obtener una mirada más global de esta problemática, de manera de incorporar como una variable importante la formación identitaria. Formación que busca interiorizarse e interiorizar a las y los estudiantes, permitirles ver y soñar un futuro en donde lo académico es tan importante como lo valórico-identitario.

Este propósito general implica conocer, describir e identificar las representaciones de las y los estudiantes universitarios en relación con permanencia/ deserción y el éxito/fracaso académico, sin embargo, las universidades optan por mirar variables o estadísticas centradas en rendimientos, comportamientos de pago o problemas sicosociales, dejando de lado el verdadero rol de la formación integral.

Para desarrollar la presente investigación, las conceptualizaciones básicas que fundamentaron este estudio provienen de la Psicología social y del análisis de contenido. La primera establece que la representación social es una manera de pensar e interpretar la realidad cotidiana, de donde se generan decisiones y acciones. Las representaciones sociales sitúan un punto de intersección entre lo psicológico y lo social, constituyendo modalidades de pensamiento práctico orientados hacia la comunicación, la comprensión y el dominio del entorno social, material e ideal. Por su parte, el análisis de contenido correspondiente a un enfoque sociológico-lingüístico del discurso para representar 
los factores relevantes que participan en la vocación, formación identitaria y éxito académico de los estudiantes,

La estructura de las representaciones implica contenidos y procesos. Los primeros son informaciones, actividades, imágenes, opiniones, reacciones y evaluaciones; para nuestro estudio, aquellas referidas al sentido y significación del éxito y fracaso académico, desde la perspectiva de los estudiantes universitarios. Los segundos remiten a la generación y transformación de lo no familiar en familiar, de aquello desconocido para el alumno en algo más concreto y manejable, más cerca de esquemas conocidos, más acompañado.

Debido a la falta de investigaciones sobre el tema, fue necesario elaborar un modelo de análisis que vinculara conceptualizaciones básicas con referentes específicos. Ello implicó integrar las representaciones de los estudiantes en relación a la institución, al plan común universidad y a los propios estudiantes. Estos elementos se conjugan, se articulan, se imbrican y entregan información variada para el reconocimiento de los contenidos y procesos de las representaciones ya que estos son atravesados por fuentes de información, contexto, actitudes y tendencias valorativas de los estudiantes universitarios.

A ello se le agregan los datos de contexto como que en Chile, de acuerdo a estimaciones basadas en estadísticas nacionales, la tasa de deserción global de estudiantes de pregrado de primer año, evaluada para el año 2010, es cercana al $30,1 \%$, siendo mayor en las universidades privadas nuevas que en las públicas. Por su parte, las áreas del conocimiento más críticas son Humanidades y Derecho con cifras del orden de $80 \%$, y las más eficientes son las áreas de Educación y Salud con un $37 \%$ y un $27 \%$, respectivamente. Por género, las mujeres poseen una tasa de deserción promedio más baja que los varones de $27,4 \%$ y $32 \%$, respectivamente (MINEDUC, 2012, p. 17-23)
Para el sistema nacional el efecto de la deserción en términos de costos es de 96,2 millones de dólares. De acuerdo a esta cifra, el gasto por abandono es equivalente al $26 \%$ del gasto público en educación universitaria, mientras que representa el $10,2 \%$ del gasto total (público y privado) en el sector universitario (UNESCO, 2004).

Dados estos antecedentes el presente artículo busca establecer una primera aproximación para conocer cuáles son las variables o condiciones desde la formación identitaria que co-ayudan a la retención y al éxito académico. Para ello se asumen dos niveles de análisis; uno propone la reconstrucción de la estructura representacional básica y compartida por los estudiantes de primer año del ingreso 2012 de la UCSH, el otro, los matices de sentido que asume la estructura representacional básica en distintos contextos institucionales.

\section{METODOLOGÍA}

La investigación cualitativa tiene como objeto conocer un fenómeno en particular de la realidad, tomando como referente principal a los sujetos, sin importar la existencia de una muestra, como lo es en el caso del método cuantitativo. Por tanto, resulta pertinente desarrollar un estudio de caso colectivo, pues "los estudios de caso colectivo implican un amplio estudio de varios casos, con el fin de permitir una mejor comprensión de lo que se conoce o quizás, una mejor capacidad para teorizar sobre un contexto más amplio" (BERG, 2007, p. 292).

En otras palabras, no importa cuántos pueden dar cuenta de un fenómeno social en particular para este ejercicio de investigación, sino el sentir de aquél que ha sido parte de esa realidad, que es protagonista de lo que sucede y se analiza, es el sustento esencial, es la referencia de una investigación de carácter cualitativo.

En ellos "se tiende a utilizar sólo enunciados de los problemas generales 
o preguntas... los problemas cualitativos tienden a ser mucho más abiertos, menos específicos, la evolución en lugar de estáticos, y orientados al proceso" (MCMILLAN, 2004, p. 49). En función del tema de esta investigación, este enfoque permitirá reconocer, a la luz del testimonio de los sujetos, impacto implícito de las configuraciones identitarias como eje articulador de permanencia/ deserción y éxito/fracaso académico, en el Programa Común Universidad (PCU) de la Universidad Católica Silva Henríquez.

El diseño metodológico se estructuró a partir del análisis comparativo intra e inter-carrera y desde la combinación de diversos procedimientos para la obtención y análisis de datos. Los procedimientos utilizados fueron tres:

a. Entrevistas estandarizadas semi-estructuradas utilizadas como técnica de recolección de datos, por cuanto se ha de implementar un listado de temas ordenados y redactadas por igual para los entrevistados, de respuesta libre y abierta para la identificación, a partir de los relatos de los estudiantes y académicos, de núcleos significativos referidos al éxito y fracaso académico.

b. Grupos Focales a estudiantes para conocer las declaraciones y percepciones más en profundidad pues la discusión y confrontación de la información percepciones, de las ideas recurrentes y/o poco comunes, de las opiniones contradictorias, y la confirmación de significados permiten conocer y resignificar lo individual en lo grupal.

\subsection{Selección de la muestra}

Se utilizó una muestra intencional y no probabilística de estudiantes universitarios de primer año, diurno y vespertino, de distintas Escuelas de la Universidad de la promoción 2012.

\subsection{Estudio y diseño}

Este proyecto se define como exploratorio, con un diseño no experimental. Se realizó según modelo de una etapa; la cualitativa. La selección de la muestra es no probabilística, de sujetos voluntarios. En esta etapa se trabajó con muestras de estudiantes universitarios de primer año de distintas careras de la UCSH.

\subsection{Recolección de datos}

Se aplicaron entrevistas semi-estructuradas y grupos focales (focus group). Se previó trabajar con seis grupos focales. Las sesiones fueron grabadas en audio, tomando notas de observación participante sólo como referencia. Y en el análisis de los datos se procedió a la construcción de categorías de acuerdo al análisis de contenido el cual se configuró como un proceso de elaboración con ajustes permanentes de principios epistemológicos, ontológicos; de estrategias analíticas; articulación de resultados; entre otros (BUENFIL, 2005).

\section{DISCUSIÓN DE LOS RESULTADOS}

Los resultados de la investigación nos permitieron confirmar la existencia de un núcleo básico, estructurante de las representaciones sobre el éxito y fracaso académico, conformado por: el propio estudiante y la institución universidad mediadas por los procesos de formación identitario.

\subsection{La autopercepción}

Como sostiene Bernal (2005), la configuración de la identidad no se plasma de manera rápida e inequívoca, si ello lo vinculamos con los estudiantes universitarios, apreciaremos que este se presenta a sí mismo como el único responsable de su situación, como el artífice de su destino académico, el que es asumido en soledad. 
Sorprende esta imagen de los estudiantes, que emerge de manera permanente y continua en el análisis de la información producto de entrevistas grupos focales, y que se traduce en la convicción de que el éxito/fracaso académico depende casi exclusivamente de su propia actuación.

Actuación que los estudiantes identifican con el aprender un oficio; el "oficio de ser estudiante", es decir; hacer y mostrar un conjunto de prácticas, actitudes, vínculos y estrategias que adquieren sentido en la medida que se adaptan y acomodan a lo esperado y valorado por los otros: Institución, profesores, compañeros. Los modos o modalidades, que los estudiantes manifiestan como necesarios para aprender este conjunto de prácticas; implican el descubrimiento, asimilación y aceptación de información tácita, rutinas ocultas, códigos y su transformación en prácticas concretas, específicas y adecuadas a las distintas situaciones por las que transita.

Lo que los estudiantes de las distintas carreras expresan como el proceso hacia el éxito académico coincidiría con lo que algunos autores denominan (COULÓN; GONZÁLEZ; CASTRO, 2007), la afiliación, es decir, la naturalización por la vía de la incorporación, de prácticas y dinámicas universitarias que no existen en los hábitos estudiantiles previos pues de un modo u otro, el sujeto precisa, en la medida de lo posible darle un sentido a su vida e inhibir el posible vacío existencial (FRANKL, 2003), el cual intenta llenar con el éxito profesional.

Nuestros resultados muestran que, los estudiantes plantean claramente que para triunfar hay que demostrar ciertas competencias que le permiten ser reconocido como miembro de la comunidad estudiantil y compartir una serie de conocimientos comunes con el resto de los estudiantes; en síntesis se trata de estructurar una nueva identidad, una nueva manera de ser, pensar y sentir. Una identidad particular, centrada en el propio sujeto y atravesada por la competencia y habilidad para desentrañar la compleja trama de reglas, normas, vínculos, prohibiciones, que los estudiantes reconocen como relevantes para el éxito académico.

Sin embargo, y como sostiene Ducoing Watty (2005, p. 100), "es a través de la acción y la palabra que el hombre se diferencia del otro". Por lo mismo, esta nueva identidad reporta un componente colectivo muy bajo, no se percibe la diferencia con el otro pues se niega, ignora $\mathrm{u}$ omite al otro, relevando que el éxito personal depende de uno mismo y que el sentido de comunidad o comunidad educativa no juega ningún rol significativo.

La soledad entonces se instala a partir de discursos desde el poder que resaltan la individualidad y que los estudiantes han asimilado muy bien, y esa ausencia de comunidad es la que los lleva al fracaso y a una difusa identidad personal, sumado a una postura clientelar de su proceso educativo, es decir, ellos van a la universidad a buscar el servicio que ésta presta que es otorgar títulos profesionales.

\subsection{La Institución Universidad}

En relación con la Institución Universidad, Facultad o carrera, entendida como una formación social y cultural compleja, en su multiplicidad de instancias, dimensiones y registros que desarrolla sus propias lógicas según la diversidad de funciones que adquiere, tanto para la sociedad en su conjunto y para los sectores sociales que las promueven y sostienen, como para los individuos singulares que son sus actores, quienes con sus prácticas cotidianas las constituyen, las sostienen y las cambian.

Esta condición quizás es una de las más esenciales pues apunta a lo central de la investigación como es la coincidencia entre las expectativas de los estudiantes con respecto a lo que la universidad declara. De acuerdo a las y los estudiantes existe un grado de coincidencia importante pues la universidad declara, para 
los estudiantes, ser diversa, pluralista, inclusiva y acogedora y en la práctica ellos lo perciben así, es decir, que se les valora, respeta, considera, acoge y ayuda a su autoconocimiento. Sin embargo, de acuerdo al discurso de las y los estudiantes la percepción de la institución es contradictoria pues desde lo que expresan y evalúan se revela con gran fuerza, el rol de los profesores.

Lo que nos permite afirmar que la institución se encarna en los profesores desde la percepción de los estudiantes, ya que son los profesores los responsables de cumplir con las funciones institucionales, especialmente, la función de evaluación y acreditación de saberes. Función vital para los estudiantes, por las consecuencias y derivaciones para el éxito y fracaso académico, que da sentido; por otra parte, a la importancia que atribuyen al conocimiento e información sobre los mismos profesores. Los estudiantes no logran hacer el nexo que es la institución la que selecciona a sus académicos de acuerdo al perfil que desean potenciar y que, por supuesto se encarna en ellos.

En lo relativo a las asignaturas del PCU (desarrollo personal, filosofía y teología) logran visibilizar más que parcialmente sus aportes en lo relativo a la formación identitaria, y aunque el programa posee un sello definido, los estudiantes sitúan ese sello no como una estrategia institucional sino como un esfuerzo de profesores y colaboradores del proceso educativo. Ello es consistente con la mirada individualista de su identidad formadora pues asocian a personas y no estrategias ni esfuerzos institucionales el sello formativo.

\subsection{La valoración de los profesores}

Se refiere concretamente a los estilos de los mismos, es decir, a sus modos de ser, actuar, orientar y regular los contenidos (no sólo conocimiento) y los procesos de enseñanza y aprendizaje. La base de la valoración de los estilos de los profesores está afincada en la cercanía, comprensión, preocupación (por el conocimiento y la realidad del estudiante), acompañamiento, facilitación del proceso de enseñanza-aprendizaje y calidad académica y personal. Esos rasgos característicos son significativos, ello sin desconocer las particularidades de cada uno, y ello le agregan que las situaciones de enseñanza se desarrollan en un ámbito grupal, exigiendo de los profesores un dominio de las "técnicas de comunicación grupal", como diría Esteve Zarazaga (2006, p. 103).

Lo anterior, las y los estudiantes lo interpretan como regulación de los procesos de éxito y fracaso académico, en la medida en que deben interpretar, resignificar y reconocer estos estilos para lograr el éxito y evitar el fracaso y donde los profesores son el nexo, el guía o la traba en la medida que no sean interpretable o sin estilo definido. La comprensión de estos estilos, su base y sus particularidades, constituirían el hilo que teje la trama de vínculos entre profesores y estudiantes; vínculo que adquiere un carácter particular en la vida del estudiante universitario y en sus representaciones sobre el éxito y fracaso académico y sobre el que afinca sus seguridades. Sin embargo, de acuerdo a nuestros entrevistados, lo central está en lo valórico del desempeño del profesor, de la acogida y el compromiso social y pedagógico que este muestra y que ellos lo ven como un esfuerzo individual y no como competencias que la institución busca en ellos.

\subsection{Acceso a información y orientación vocacional}

Los estudiantes plantearon en los grupos focales y entrevistas la necesidad de aumentar y mejorar la información sobre las carreras, no sólo a nivel de planes de estudios o mallas curriculares, sino también en forma especial, aquella relacionada con orientación vocacional y 
nivelación de competencias. La información es importante, pero la orientación antes de postular es fundamental para disminuir brechas entre exigencias de las carreras y las competencias de los postulantes. Aun después de ingresar a las carreras en las universidades, los estudiantes continúan requerimiento ayuda de orientación, apoyo psicológico y apoyo académico, según destacaron en sus discursos.

Por el lado de los directivos universitarios, éstos reconocen que, en general, no existen estrategias tan definidas de retención de alumnos vulnerables, ya que el énfasis de los registros está en el seguimiento del rendimiento académico, el cual se usa ocasionalmente para realizar seguimiento a los estudiantes con problemas académicos.

Si se destaca que la institución posea instancias de nivelación de capital cultural o competencial, aunque estos esfuerzos sean reducidos y focalizados en los estudiantes más vulnerables.

\subsection{Ausencia de la noción carrera y plan de estudios}

Así como mencionamos la dispar mirada sobre la Institución en las representaciones de los estudiantes, debemos referirnos también a la Ausencia de la carrera y del plan de estudios, los que se encarnan o se perciben mediante las materias y/o contenidos específicos, desde la adhesión o el rechazo (por las dificultades que genera en algunos casos) que estas provocan. En las representaciones de los estudiantes el plan de estudios consiste en materias en secuencia que hay que pasar y/o aprobar. El estudiante y la Institución - facultad -profesor - contenidos- como dimensiones estructurantes de las representaciones sobre el éxito y fracaso académico se desagregan en núcleos temáticos dispersos, no integrados, es decir, las y los estudiantes no logran ver la globalidad, no integran, no ven secuencialidad.
En la medida que conciba a su carrera y a la formación identitaria, articulada a través del plan común, como un conjunto disgregado de asignaturas y además con ello, suponiendo que muchas de ellas no se justifican, entorpecerá su desarrollo, su comprensión y su motivación que le ayudarán a su éxito académico.

\subsection{Contextos tensionantes del éxito o fracaso académico}

Si partimos de la base que la realidad social no tiene "esencias eternas, estáticas e inamovibles, sino fenómenos en movimiento, oscilan en el péndulo de la tradición y el cambio y ambas posibilitan un acercamiento al tejido sociocultural para una mejor comprensión de lo que somos y de lo que podemos lograr" (GIMÉNEZ, 2007, p. 9), entonces, las condiciones del éxito o fracaso académico también son dinámicas. El éxito y fracaso académico está referido a un conjunto de aspectos personales, sociales, económicos y culturales. Estos aspectos se amalgaman en dos miradas coincidentes, que todos las y los estudiantes y también los profesores manifiestan como inherente o propio del "ser estudiante" y como necesario para alcanzar el éxito.

Estas condicionantes contextuales son percibidas de manera diferente por las y los distintos estudiantes pues sus marcos de realización están mediados por los contextos de origen, su formación escolar, su proyecto de vida y sus experiencias vitales. Para algunas y algunos estos contextos son la gatillante de la superación, para otros la condicionante para un mejor rendimiento o para otros una presión extra que termina por excluirlos del sistema. Más allá de las distintas percepciones, los contextos en los que se desenvuelven en su vida académica y en la proyección de contextos profesionales futuros estarán influidos por su contexto de origen. Ello implica que, de acuerdo a los discursos de los estudiantes, contextos 
más empobrecidos económica y culturalmente son más proclives a fracaso académico y a una estructuración identitaria más difusa o más resistida.

\subsection{Temporalidad del estudio}

El tiempo dedicado al estudio, tal como lo valoran las y los estudiantes de las distintas carreras que cursan asignaturas en el plan común, a simple vista nos revela una cuestión de cantidad, cantidad de horas de las que el estudiante dispone y realmente ocupa para "estudiar". Si trascendemos esta primera idea cuantitativa y vamos más allá de las percepciones y manifestaciones de los propios estudiantes; podemos pensar que el tiempo, como el espacio, aparece a la vez como un elemento natural y como una estructura simbólica que pone ritmo en la vida individual y en la social.

El tiempo, tal como se revela en las representaciones de los estudiantes y de acuerdo con lo que sostienen Marc y Picard (1992), constituye una de las dimensiones esenciales de la relación del hombre con su entorno y con sus semejantes, marca profundamente sus ocupaciones, sus ritmos diarios, su futuro y su concepción de la vida. La temporalidad es percibida como reguladora de la interacción entre las y los estudiantes y entre las y los estudiantes y los profesores.

Nuestros resultados nos muestran que la noción de tiempo se asocia directamente con lo que se dedica al estudio, tiempo y estudio parecerían sinónimos o expresiones intercambiables en la percepción del estudiante y también en la de los profesores. Pero en los discursos aparece también la noción de tiempo segmentado, es decir, muchas y muchos estudiantes no poseen una regularidad temporal de estudio sino que es aleatorio, ya sea en las horas o en los días a dicha función.

\subsection{Conocimiento del contexto evaluativo}

Es otra de las condiciones que forma parte de las representaciones sobre el éxito académico. Tal como se desprende de los análisis realizados; este saber se conforma por distintos tipos de conocimientos que la o el estudiante afirma que debe "aprehender" y que nos permite definir sus significados. En este saber de los estudiantes identificamos:

a) Información referida al Profesor que el estudiante debe descubrir; que le gusta, que no le gusta, cómo pregunta y le gusta que le respondan, como hay que dirigirse, entre otras cosas.

b) El saber referido a lo que el estudiante debe aprender a hacer y a cómo debe actuar en función de lo que conoce del profesor. Pero, ¿por qué el saber rendir exámenes constituye una condición para el éxito? Esta interrogante lleva a reconocer algunas fuentes para su explicación e interpretación, como es el caso de los planteos de Foucault (1977, p. 185), cuando afirma que "la superposición de las relaciones de poder y de saber asumen en el examen su máximo esplendor".

El examen es un permanente "intercambiador de saber", podríamos agregar entre el profesor y el estudiante; entre los estudiantes; entre el estudiante y la propia comunidad. En nuestro estudio uno de nuestros focos es el intercambio profesor-estudiante, porque justamente es el primero, el que a través del examen legitima, aprueba o desaprueba el valor de estos saberes; tal como lo manifiestan los estudiantes. Es necesario destacar que en la percepción del estudiante, el profesor no es responsable de los resultados de un examen. Lo que sí es clave, tanto como el conocimiento que se intercambia en los exámenes; el saber rendirlos.

Estos resultados nos permiten afirmar que tanto el contenido, como el procedimiento en los exámenes; el qué y 
el cómo hacerlo, son parte importante de las representaciones de las y los estudiantes. En algunos casos lo determinante es el procedimiento, es el cómo. Vale tanto saber rendir un examen, que saber sobre lo qué se va a rendir.

\section{ESTRATEGIAS Y PRÁCTICAS}

Las estrategias se refieren a un conjunto de procedimientos, tareas para la consecución de una meta o fin y las prácticas son acciones específicas, consecuencia en algunas casos de un accionar estratégico y en otros, de la propia situación que vive la y el estudiante; la cual puede llevarlo a realizar actividades no planificadas con anterioridad o a decidir de forma inmediata acciones puntuales. Al referirnos a las estrategias que los estudiantes ponen en juego, debemos reconocer que toda Institución tiende a inducir una regulación y una codificación de las relaciones sociales que se traducen en una ritualización de la comunicación; rituales o ritos como códigos de conductas que presiden las interacciones sociales cotidianas (MARC; PICARD, 1992, p. 106).

Estos códigos, que los estudiantes lo llaman reglas, normas o simplemente algo que hay que cumplir son interpretados por ellos en distintas situaciones de interacción que viven cotidianamente: clases, exámenes, encuentros con profesores, participación en clase, relaciones con compañeros, pedidos de información a personal de la Institución, búsqueda de material de estudio; para conocer cómo se actúa habitualmente, qué se permite, qué se prohíbe, qué se deja pasar. Todo este cúmulo de información se va asimilando justamente a medida que se participa en las situaciones mencionadas; es decir, que parte de los códigos, en nuestro caso códigos universitarios, se develan en la propia acción, en el momento en que el estudiante está actuando, de ahí la complejidad de este proceso de descubrimiento.
Complejidad que los estudiantes expresan como dificultad para reconocer y diferenciar rápidamente en qué clase estoy, con qué profesor, cómo debo actuar, que define al profesor y que debo evitar. Para algunos estudiantes resulta algo sencillo y "rápido" de asimilar, para otros más difícil de aprehender y comprender lo que pasa y se juega en cada situación académica. En cualquiera de los dos casos, nuestros resultados del estudio nos muestran que los estudiantes deben aprehender a reconocer, interpretar y re-significar de manera permanente el tipo de situación en la que están inmersos, la información que necesitan para actuar; y luego decidir la forma de actuación más conveniente.

Por ejemplo, la participación en clases, es una práctica reconocida por los estudiantes como necesaria para el éxito. Es importante participar en las clases, dicen las y los estudiantes, pero es una práctica condicionada; por el profesor, por el propio desarrollo de la clase, por el tema, por la forma de hacerlo - participación breve y puntual o una larga intervención. Y justamente en estos aspectos que condicionan la participación es donde el estudiante debe actuar de manera estratégica: evaluar la situación, confrontar con la información que dispone y decidir qué hacer.

En el caso de las asignaturas del plan común éstas tienen su centro en la participación activa de la y del estudiante, y ellos lo perciben de esta manera pues declaran que es un espacio para compartir experiencias, encontrarse con ellos mismos, mirar sus posibilidades de futuro y compartir un espacio identitario común.

\subsection{Actitudes}

Las actitudes se refieren a una predisposición permanente de un individuo a reaccionar en un sentido sea cual fuere la situación, es una constante del comportamiento. Las actitudes adquieren un peso 
muy importante en las representaciones sobre el éxito y fracaso académico; repitiendo lo anterior es una constante en la información obtenida tanto en los grupos focales y las entrevistas.

Constante presente en las y los estudiantes y en las y los profesores. Lo interesante respecto a las actitudes es la manera en que se manifiestan en los resultados obtenidos: ¿qué es lo más importante cuando de actitudes se trata?: "evidenciarlas" como algo que se ejecuta sin necesidad de que forme parte del comportamiento de una y un estudiante o asumirlas como propias de la personalidad de cada estudiante.

Las actitudes están en el centro de lo identitario, y las y los estudiantes comprenden que ellas les permitirán avanzar o detenerse. Lo interesante de las entrevistas y focus es que la responsabilidad y el compromiso con sus estudios es una constante entre estudiantes, ello está influenciado por ser casi el ciento por ciento primera generación en la universidad y el fracaso y decepción hacia la familia es un potenciador, en la mayoría de los casos, de actitudes resilientes

\subsection{Ser o parecer que se es}

Como expresan las y los propios estudiantes: "ser responsable, dedicado, constante o mostrarse con estas cualidades en determinados momentos". Se destaca desde el principio esta cuestión, por la recurrencia de lo actitudinal y por la manera en que es percibida por los estudiantes. Las actitudes tienen que ver para los estudiantes, con el poder llevar la carrera al día, con el estudio, con los consejos que darían a otros estudiantes, con los exámenes, con las razones y condiciones del éxito académico, con las posibilidades de evitar el fracaso, con las relaciones y vínculos que se establecen, con la propia identidad del estudiante, con las metas y expectativas futuras.

Las actitudes que resultan más importantes, desde las representaciones de las y los estudiantes, se presentan en orden jerárquico y son: actitudes personales (responsabilidad, compañerismo, honestidad, entre otras), relacionadas con el estudio y con el manejo del tiempo y sobre todo, compromiso familiar.

\subsection{Establecimiento de vínculos}

Los más importantes y significativos en las representaciones sobre el éxito y fracaso académico son los que se establecen con los profesores. Vínculos o relaciones unidireccionales, en el sentido de la aceptación de las reglas y normas que imponen los profesores, por una parte, $\mathrm{y}$ del acogimiento, respeto, preocupación, apoyo, por otro.

En menor medida se valoran los vínculos con los compañeros, lo cual no significa que no existan, sino que cuando del éxito y fracaso se trata no adquieren gran importancia. Esto es coherente, con lo que ya venimos planteando en cuanto a que los profesores, de acuerdo a lo expresado por las y los estudiantes, son los reguladores del éxito y fracaso de los estudiantes y los vínculos que puedan establecerse con ellos son vitales en este proceso de regulación y control.

\section{PROPUESTAS Y PROYECCIONES}

Si tomamos en conjunto los aspectos desarrollados podemos afirmar que el éxito académico, desde las representaciones de los estudiantes, se define fundamentalmente como un "proceso adaptativo" o un proceso de adaptación que el estudiante debe asumir al ingresar a una facultad, acompañado de diversas reglas que se deben conocer y saber manejar adecuadamente, a la manera de un juego que se va definiendo al mismo tiempo que se lo juega.

La formación identitaria está asociada de forma significativa, sobre todo con enfoques profundos y estratégicos, así como con las expectativas de los 
alumnos sobre el proceso de la enseñanza y sus resultados no sólo a conocimiento sino que también a valores como honestidad, empatía, compañerismo, respeto o acogimiento se encuentran asociados a enfoques profundos y estratégicos y a expectativas de los alumnos sobre el aprendizaje, sus resultados y su propio desarrollo personal y profesional.

Ahora, y de acuerdo con los discursos de las y los estudiantes parece que los valores no tienen muchas relaciones directas y fuertes con la satisfacción con los estudios, sin embargo, sí parecen estar muy relacionados con los determinantes más relevantes del rendimiento académico pues en la medida que ven satisfechas todas o muchas de sus expectativas valóricas (imaginario de los y las estudiantes), a las cuales aporta el plan común, ello las y los seguriza, motiva, disminuye ansiedad y les permite reconocerse como otro en relación a otros que viven el mismo proceso.

Los estudiantes sólo descubren el alcance y la aplicación de las reglas en situación, esto es, al ponerlas en práctica. Las reglas se definen por su uso. Esta idea es el primer principio o regla que el estudiante debe aprender sobre las reglas mismas. Se puede saber como actuar o no, pero este saber ser se interpreta y modifica en las distintas situaciones en las que se actúa. El carácter adaptativo del éxito, el rol "pasivo" del estudiante, en esta adaptación y las reglas que deben desentrañarse en el propio juego institucional encarnada casi con exclusividad por los profesores constituyen la trama central o la estructura básica compartida de las representaciones sobre el éxito y su contraparte, como otra cara, no necesariamente opuesta, el fracaso académico; por los estudiantes de las distintas carreras.

Precisamente, el estudio de las representaciones sociales es pertinente y valioso desde nuestra perspectiva para aproximarnos al estudio de la identidad, lo que será factible a partir de su articulación desde una perspectiva histórica con una visión actual y futura. Será importante, también, generar seguimientos de estos egresados, a fin de conocer las formas en que sus representaciones sociales que ellos reporten se modifican o reafirman en función de su inserción laboral, en un contexto en el cual se está dando en general una dificultosa y tardía incorporación al mercado laboral de los jóvenes, cuando ocurre, y que cuestiona el proyecto de vida al que aspiran las nuevas generaciones

Finalmente, de acuerdo con lo anteriormente expuesto, se concluye que los principales factores asociados al rendimiento académico de las y los estudiantes encontrados en la presente investigación se refieren a las expectativas del alumno, las expectativas de su entorno personal, sus habilidades sociales y todo el aporte de acompañamiento y formación identitaria que la universidad, vehiculizado a través de sus profesores, es capaz de entregar, los cuales en la relación que manifiestan, tienen la probabilidad de predecir la retención y el éxito académico.

\section{REFERENCIAS}

BERG, B. Qualitative Research Methods for the Social Sciences. Sixth Edition. Boston: Pearson A. \& B., 2007.

BERNAL, A. Reconceptualización de la Identidad Personal y Educación para la Autodeterminación Posible, Teoría de la Educación. Revista Interuniversitaria, n. 17, p. 97-128, 2005.

BUENFIL, R. N. Notas Personales Tomadas en el Marco del Seminario Interinstitucional (FFyL-Unam/DIE-Cinvestav) "Programa de Análisis Político de Discurso e Investigación", PAPDI, México, 2005.

COULÓN, A.; GONZÁLEZ, M. A; CASTRO, F. Los desertores del futuro. Córdoba, Argentina: Centro de Estudios Avanzados, Universidad Nacional de Córdoba, 2007.

DUCOING WATTY, Patricia (Coord.). Sujetos, Actores y Procesos de Formación. México: COMIE, 2005. (Col. La investigación Educativa en México 1992-2002, tomo II). 
ESTEVE ZARAZAGA, J. M. Las Emociones en el Ejercicio Práctico de la Docencia, Revista Teoríade la Educación, n. 18, p. 85-107, 2006.

FRANKL, V. El hombre en busca de sentido. Barcelona: Herder, 2003.

GIMÉNEZ, Gilberto. Estudios sobre la Cultura y las Identidades Sociales. México: Consejo Nacional para la Cultura y las Artes/ITESO, 2007.

MARC, E.; PICARD, D. La Interacción Social. Cultura, Instituciones y Comunicación.
Buenos Aires: Paidós, 1992.

MCMILLAN, J. Educational Reserch, Fundamentals for the Consumer. 5. ed. EEUU: Pearson Editorial, 2004.

MINEDUC. Deserción en la educación superior en Chile. Santiago, Chile: Centro de Estudios MINEDUC, 2012.

UNESCO. Educación Superioren una Sociedad Mundializada. Francia: UNESCOEDUCACIÓN, 2004.

\section{Sobre o autor:}

Carlos Mejías Sandia: Doctor en Sociología. Profesor de Lenguaje. Académico e investigador de la Universidad de Talca, Chile. E-mail: camejias@utalca.cl 